\title{
Efficacy of mesenchymal stem cells in treating patients with osteoarthritis of the knee: A meta-analysis
}

\author{
GANG-HUA CUI, YANG YANG WANG, CHANG-JUN LI, CHEN-HUI SHI and WEI-SHAN WANG \\ Department of Orthopedics, Medical College of Shihezi University, Shihezi, Xinjiang 832008, P.R. China
}

Received June 22, 2015; Accepted August 16, 2016

DOI: 10.3892/etm.2016.3791

\begin{abstract}
To assess the clinical efficacy and safety of mesenchymal stem cell (MSC) treatment for osteoarthritis of the knee (KOA), a systematic electronic literature search was performed on PubMed, EMBASE and Web of Science. Studies published in English from the earliest record to December 2014 were searched using the following keywords: Cartilage defect, cartilage repair, osteoarthritis, KOA, stem cells, MSCs, bone marrow concentrate (BMC), adipose-derived mesenchymal stem cells, synovial-derived mesenchymal stem cells and peripheral blood-derived mesenchymal stem cells. The effect sizes of selected studies were determined by extracting pain scores from the visual analog scale and functional changes from International Knee Documentation Committee and Lysholm and Western Ontario and McMaster Universities Osteoarthritis Index before and after MSCs or reference treatments at 3, 6, 12, and 24 months. The factors were analyzed and the outcomes were modified after comparing the MSC group pooled values with the pretreatment baseline or between different treatment arms. A systematic search identified 18 clinical trials on this topic, including 10 single-arm prospective studies, four quasi-experimental studies and four randomized controlled trials that used BMCs to treat 565 patients with KOA in total. MSC treatment in patients with KOA showed continual efficacy for 24 months compared with their pretreatment condition. Effectiveness of MSCs was improved at 12 and 24 months post-treatment, compared with at 3 and 6 months. No dose-responsive association in the MSCs numbers was demonstrated. However, patients with arthroscopic debridement, activation agent or lower degrees of Kellgren-Lawrence grade achieved improved outcomes. MSC application
\end{abstract}

Correspondence to: Professor Wei-Shan Wang or Professor Chen-Hui Shi, Department of Orthopedics, Medical College of Shihezi University, 361 Beisi Road, Shihezi, Xinjiang 832008, P.R. China

E-mail:wwsmc2002@sina.com

E-mail: gksch7890@sina.com

Key words: mesenchymal stem cells, knee, osteoarthritis, articular cartilage, stem cell therapy, meta-analysis ameliorated the overall outcomes of patients with KOA, including pain relief and functional improvement from basal evaluations, particularly at 12 and 24 months after follow-up.

\section{Introduction}

Osteoarthritis (OA) is a chronic, progressive and degenerative joint disease, involving single or multiple joints. OA of the knee (KOA) is the most common disabling disease, characterized by the degeneration and degradation of cartilage, subchondral bone remodeling, osteophyte formation and synovial inflammation, which affects the patient's quality of life and constitutes a heavy financial burden (1-3). With the exception of oral and intra-article injection medications that relieve the symptoms and improve joint function, there is no approved medical treatment that halts disease progression and joint destruction $(1,4)$.

Various surgical methods, including microfracture $(5,6)$ and subchondral drilling (7), have been proposed to regenerate articular cartilage. However, due to the complications and inferior quality of the regenerative fibrocartilage, risky and cost-effective joint replacement surgery is often ultimately required (8). Previous studies have investigated tissue engineering and cellular therapies for treating early stage OA, and autologous chondrocyte implantation has demonstrated positive clinical outcomes $(9,10)$. Nevertheless, due to the poor self-renewal and regeneration potential of chondrocytes, it is a slow process that may lead to fibrocartilage rather than hyaline cartilage $(11,12)$. Furthermore, this two-stage surgical procedure and is predominantly used to treat cartilage defects caused by injury rather than OA.

Therefore, research attention in this field has shifted to the more promising treatment of mesenchymal stem cells (MSCs). MSCs, which can be derived from blood, bone marrow, skeletal muscle, adipose, skin and synovial membrane (13), have the capacity to differentiate into osteocytes, adipocytes, chondrocytes, myoblasts, tenocytes $(14,15)$, secrete bioactive molecules that stimulate angiogenesis and tissue repair, and reduce the response of T cells and inflammation $(16,17)$. Previous clinical trials have reported that mild/moderate OA or advanced OA can be treated efficiently using autologous or allogenic MSCs through implantation, micro fracture or intra-articular injections (18-20). However, so far, no meta-analytic research has evaluated the efficacy and safety of MSCs in treating patients with KOA. 
Therefore, the present meta-analysis was conducted to analyze the clinical outcomes of MSC treatment on patients with KOA patients by analyzing pain and functional changes, compared with their pretreatment condition, or placebo controls.

\section{Materials and methods}

Search strategy and eligibility criteria. Electronic databases: including PubMed (ncbi.nlm.nih.gov/pubmed), EMBASE (embase.com) and Web of Science (webofknowledge.com), were used to comprehensively search for all relevant studies published in English from the earliest record to December 2014. The following keywords were used: 'cartilage defect', 'cartilage repair', 'osteoarthritis', 'knee osteoarthritis', 'stem cells', 'mesenchymal stem cells' (MSCs), 'bone marrow concentrate', 'adipose-derived mesenchymal stem cells' (ADMSCs), 'synovial-derived mesenchymal stem cells' and 'peripheral blood-derived mesenchymal stem cells', as medical subject headings or text words. In addition, Cochrane Systematic Reviews (cochrane.org/evidence) and ClinicalTrials.gov were manually searched for additional references. Articles were considered eligible if they met the following criteria: i) Patients were $\geq 18$ years-old and had KOA symptom or diagnosed with KOA by clinical and imaging examination; ii) MSCs administered to at least one treatment group; iii) $\geq 3$-month follow-up; iv) $\geq 1$ valid outcome measurement before and after the administration of MSCs, such as the visual analogue scale (VAS), International Knee Documentation Committee (IKDC) Subjective Knee Form, Lysholm scale, and Western Ontario and McMaster Universities Osteoarthritis Index (WOMAC); and v) outcomes were presented as continuous data [mean \pm standard deviation (SD)]. Studies that lacked an intervention plan or pain and functional measurements were excluded.

Data extraction and study quality assessment. Two independent reviewers searched the electronic databases and evaluated the eligibility of the searched articles and subsequently extracted data using a standardized form, including data on the study type, number of patients enrolled, patient characteristics, disease duration, dosage of MSCs, outcome measurements, follow-up time and adverse events. If additional data was necessary, the authors were contacted for further information. The Jadad scoring system was used to assess the methodological quality of the randomized controlled trials (RCTs) (21). The quality of the included RCTs ranged from $0-5$ points, with a score of $<3$ indicating a low-quality study. The Newcastle-Ottawa Scale (NOS) was used to assess the quality of other studies according to selection, comparability, exposure, and outcome, including single-arm prospective and quasi-experimental studies (22). NOS was scored out of 9 points, with total scores $<4$ points defined as low quality. Discrepancies between the two independent evaluations of potential articles were resolved by discussion and consensus.

Data synthesis and analysis. Data were extracted from four time points at or closest to the 3rd, 6th, 12th and 24th months after MSCs treatment. Effect size (ES) was calculated

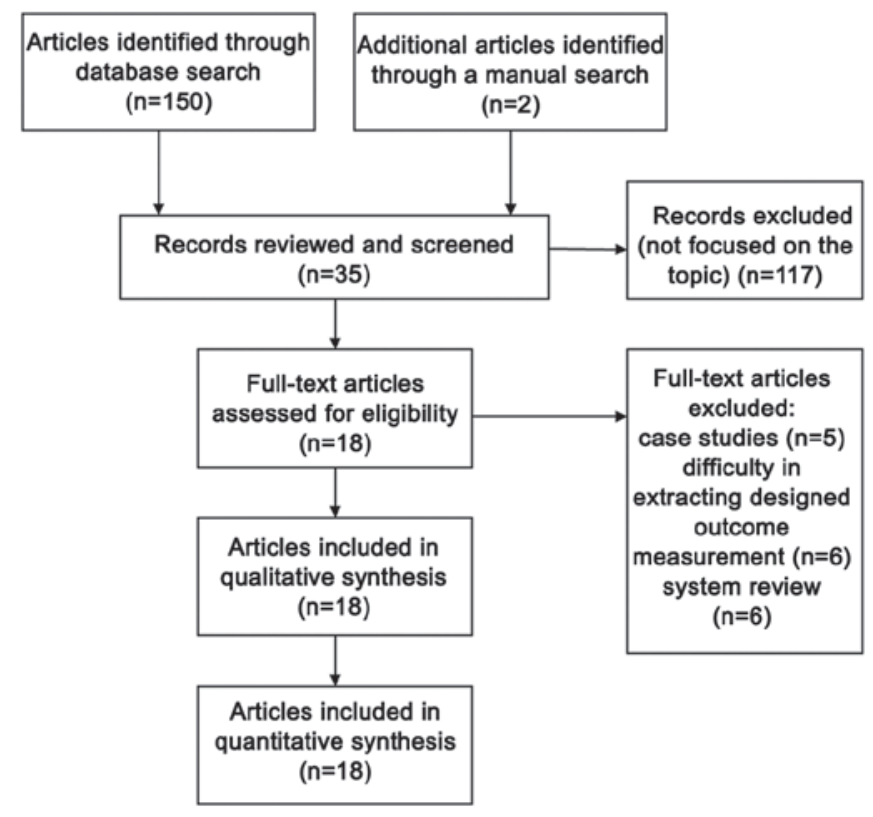

Figure 1. Flow chart of the evaluation process for the inclusion or exclusion of studies.

from knee joint pain and functional changes and the results were compared with the pretreatment baseline or between different treatment arms. VAS was extracted from the included articles. If $>1$ functional measurement was included in an article, only one functional scale in line with the order of IKDC, Lysholm and WOMAC was chosen. As multiple treatment groups wew included in some articles, each group was selected as a separate status set to analysis. Mean \pm SD between the pretreatment baseline condition and functional scores after treatment was used to evaluate the effectiveness of MSCs therapy. Positive ES values demonstrated a pain or functional improvement, and vice versa. For studies in which the measurement score and SD was deficient, the value was calculated from the P-value of the corresponding hypothesis test. If the measurement scores and SD could not be extracted in some articles, a correlation of 0.5 was used to estimate the dispersion. A random effect model was used to pool the ESs with a $95 \%$ confidence interval $(95 \% \mathrm{CI})$ on the basis of heterogeneity. A positive pooled ES with a 95\%CI $>0$ indicated an advantage of MSCs compared with the pretreatment condition or reference treatments.

Assessment of heterogeneity and sensitivity. Statistical heterogeneity was assessed via the I-square and Cochran's Q tests. A P-value of $<0.10$ for $\chi^{2}$ test or an I-square $>50 \%$ was indicative of the existence of substantial heterogeneity (21). Subgroup analysis was performed according to variables of the study design, different dosages, arthroscopic debridement (AD), activation agent, as well as the severity of Kellgren-Lawrence (K-L) grades. Sensitivity analysis was performed by excluding some articles with extreme ES values to assess whether the movement resulted in serious changes in the total result. Funnel plots were used to assess the potential publication bias. All analyses were conducted using Review Manager Version 5.2 (The Cochrane Collaboration, Oxford, UK). 


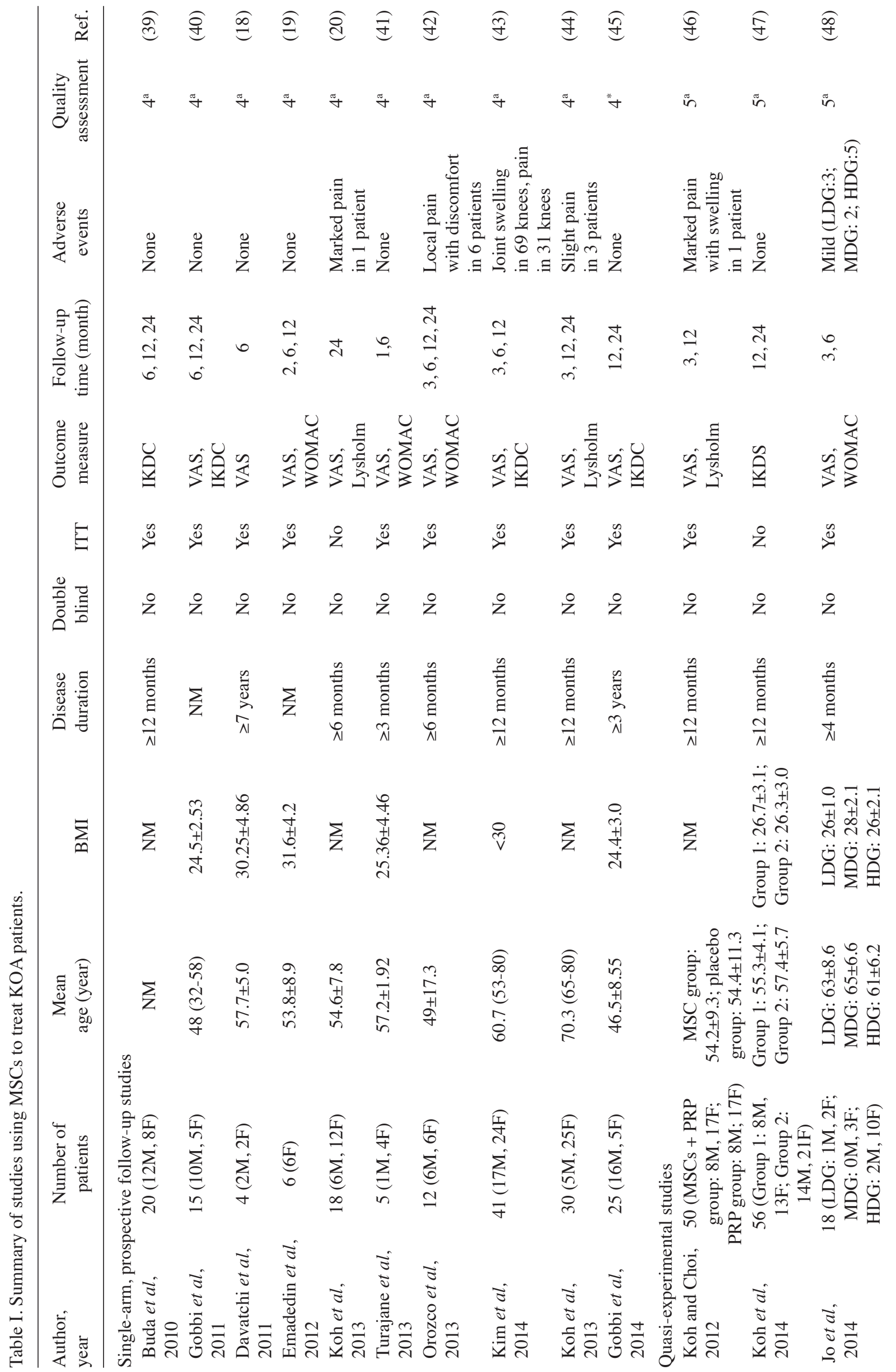




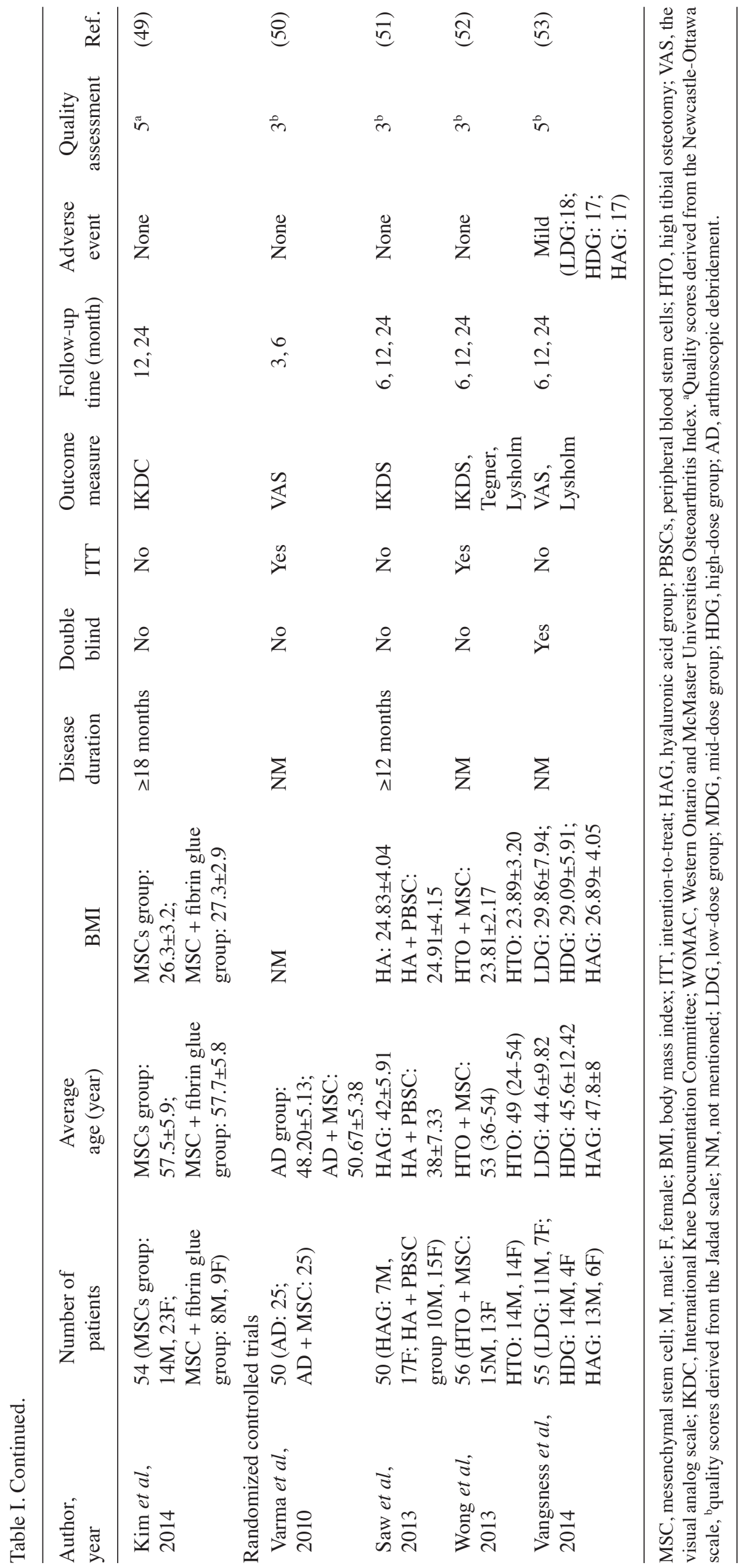




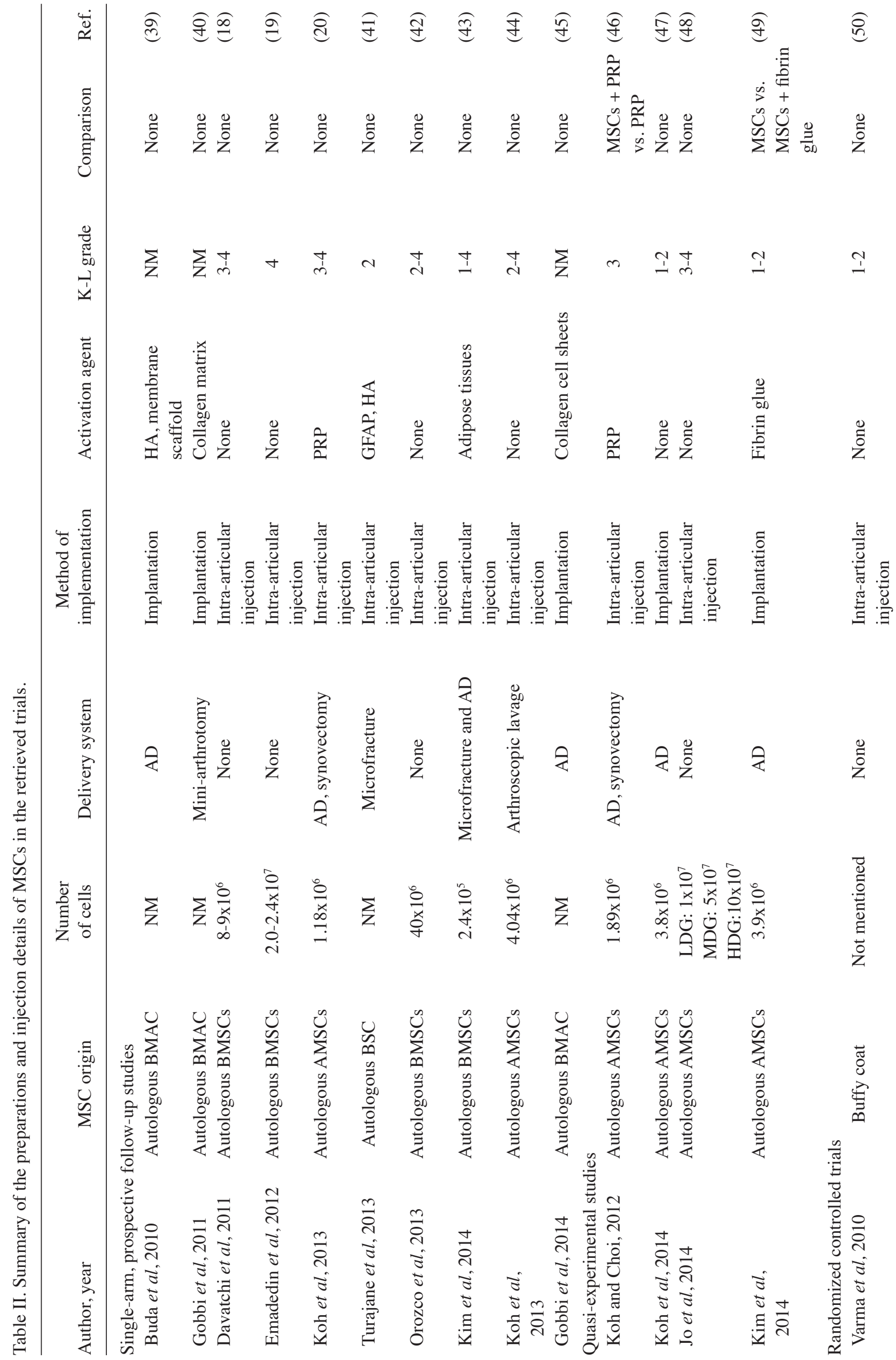




\section{Results}

Study characteristics. A total of 152 studies were initially searched, of which 117 were removed after title and abstract screening. Of the 35 citations, 18 clinical studies which met the inclusion criteria were identified for eligibility (Fig. 1); five case studies (17,22-26) were excluded and nine studies $(24,27-34)$ were removed due to difficulties in extracting the outcome measurements. Four systematic reviews (35-38) were also excluded. An assessment of the remaining 18 studies revealed that 10 used a single-arm prospective design (18-20,39-45), four used quasi-experimental trials (46-49) and four used RCT (50-53) (Table I). A total of 565 participants (226 males and 339 females) were included from the 18 studies. The duration from the onset of knee pain to registration in each study was 3 months to $\geq 7$ years. The follow-up period was 3-24 months. The majority of studies recruited patients with KOA with a severity grade of 1-4 on the K-L scale. K-L grade s 1-2, and grades 3-4 were defined as early OA and advanced OA, respectively (Table II).

Effects of MSCs. Compared with the pretreatment condition, a pooled ES of $0.80(95 \% \mathrm{CI}, 0.42-1.17)$ was determined at 3 months, 1.72 (95\% CI, 1.13-2.31) at 6 months, 2.03 (95\%CI, 1.30-2.76) at 12 months (Fig.2), and 1.81 (95\% CI, 1.62-2.00) at 24 months (Fig. 3), which all favored the status after MSCs treatment. Following the exclusion of an outlier with an extremely high ES, the beneficial effects from MSCs treatment remained, with an ES of 0.77 (95\% CI, 0.41-1.13) at 3 months, 1.49 (95\% CI, 0.93-2.04) at 6 months, 1.63 (95\% CI, $0.99-2.27)$ at 12 months, and $1.74(95 \% \mathrm{CI}, 1.55-1.93)$ at 24 months. A significant superiority of MSCs intervention was demonstrated by a high summed ES at 12 and 24 months without an overlap of the 95\% CI of ES at 3 months, which indicated that the treatment effect of MSCs on KOA patients improved significantly over time. However, after excluding the data from quai-experimental and single-arm prospective studies and only using the data from RCTs, the treatment of MSCs did not demonstrate superiority. Relative to the baseline, patients improved in the pain and functional scale scores at all time points.

Stratified analysis. Participants receiving MSC treatment were stratified according to the study design, administration dosage, AD, activation agents and K-L grades. Point estimates of the pooled ES in the single-arm prospective studies and quasi-experimental trials were higher than those in the RCTs, and an uncertainty in the treatment effectiveness emerged regarding participants in the RCTs at 6,12 and 24 months, since the $95 \% \mathrm{CI}$ of the summed ES crossed the value of 0 . Stratified analysis failed to demonstrate a dose-responsiveness association in the MSC numbers. However, the treatment effectiveness in the MSC groups with AD or activation agents was superior to the MSC groups without AD and activation agents, particularly at 12 months in the activation agents group (ES, 3.13; 95\% CI, 1.55-4.71) compared with the group without activation agents (ES, 0.67; 95\% CI, 0.01-1.34). And the early OA group exhibited a higher ES point estimate at all time points than the advanced OA group (Table III). 
A

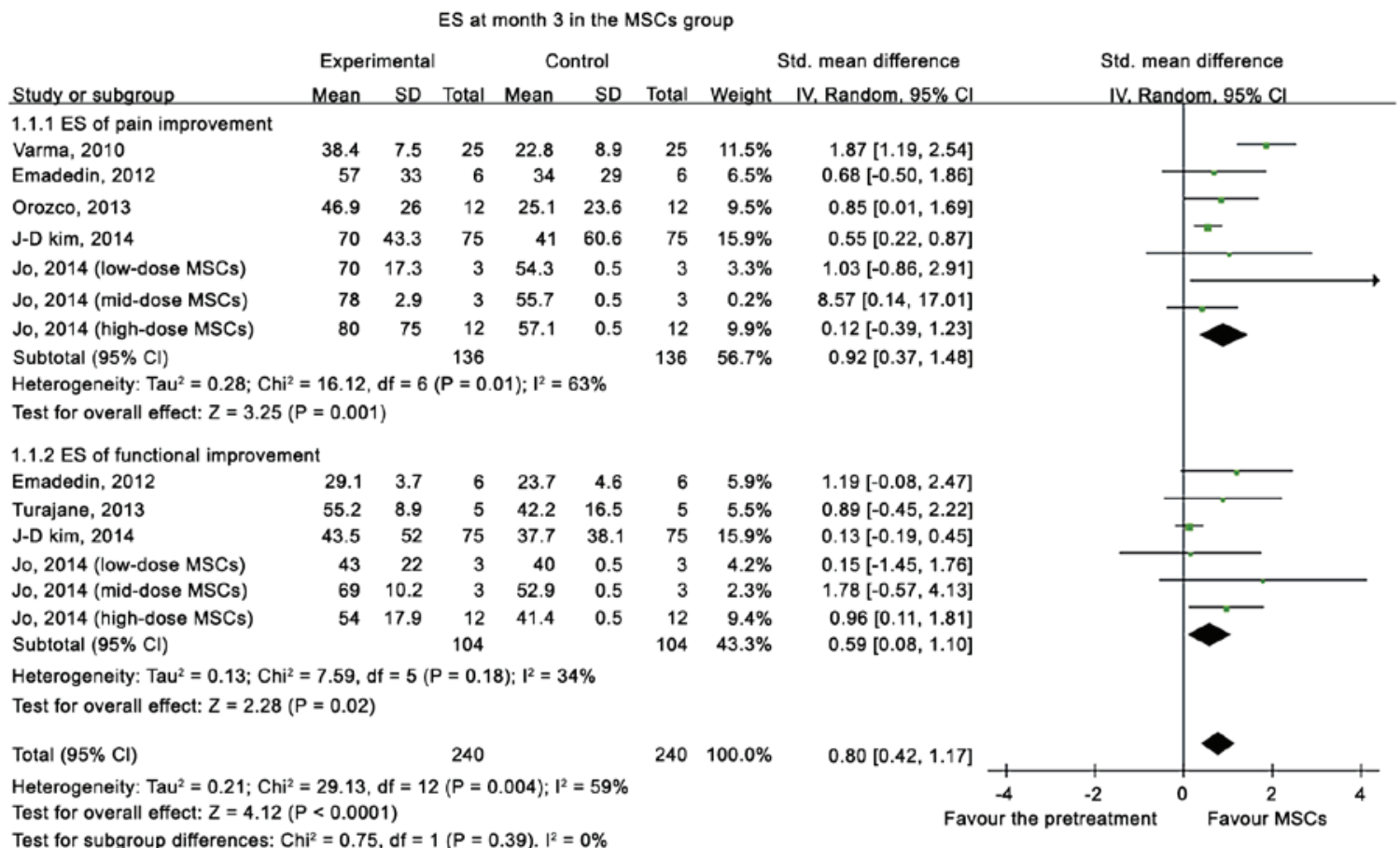

B

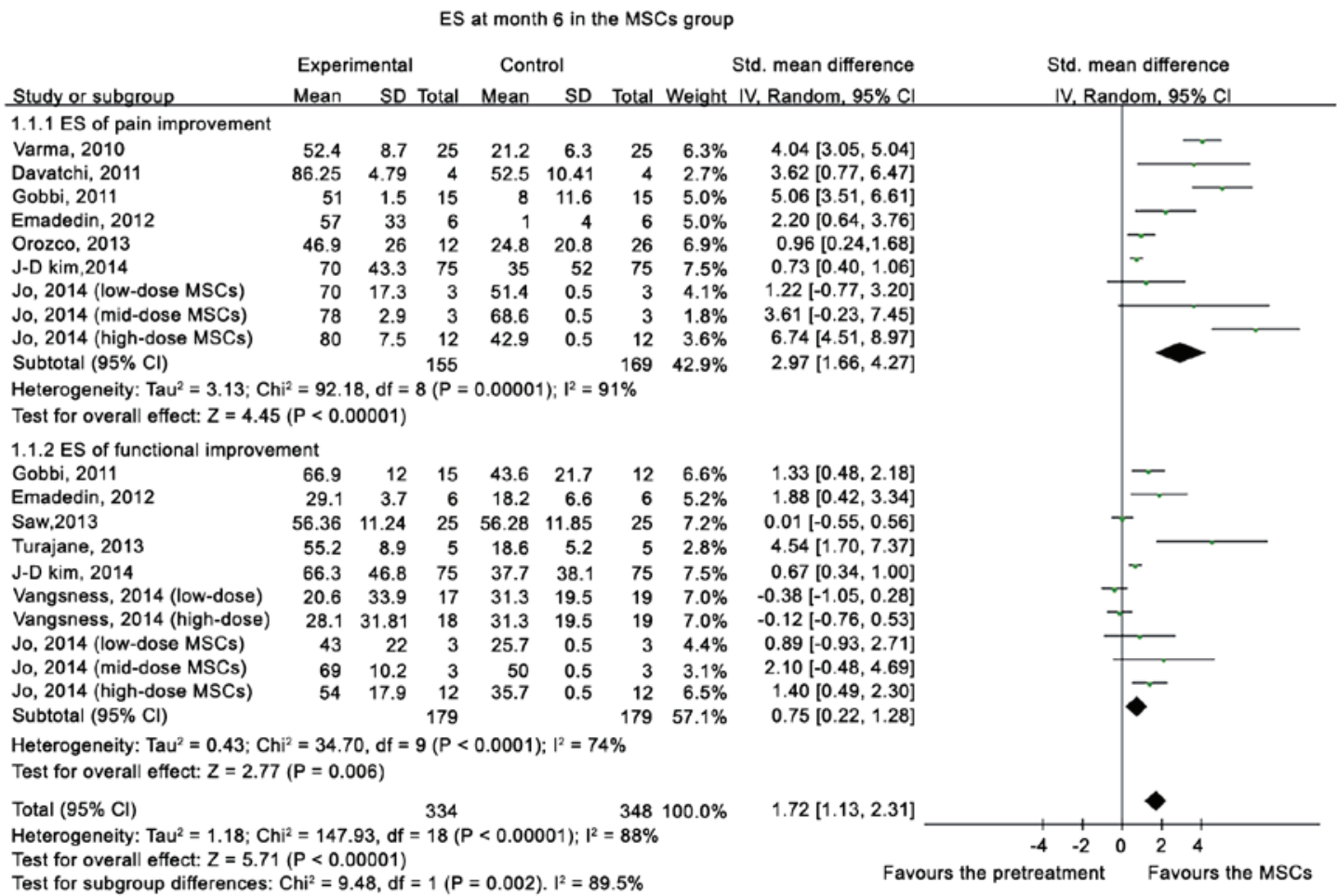

Figure 2. Forest plot of ES of pain and functional changes from baseline at (A) 3 and (B) 6 months after MSC treatment. ES, effect size; MSC, mesenchymal stem cell; SD, standard deviation; IV, inverse variance; CI, confidence interval.

Adverse effects and publication bias. Seven of the 18 trials reported adverse events after MSC treatment, in which the predominant symptoms were local swelling and transient regional pain. All of the adverse events reported by patients were self-limited or were remedied with therapeutic measures. None of the patients included in the present study were diagnosed with cancer that was associated with MSC therapy. Asymmetry was observed in the funnel plots based on the ESs of changes in the pain and functional scales from baseline (Fig. 4).

\section{Discussion}

The present meta-analysis comparing the conditions of patients with KOA before and after treatment with MSCs demonstrated a continual efficacy for at least 24 months. 
A

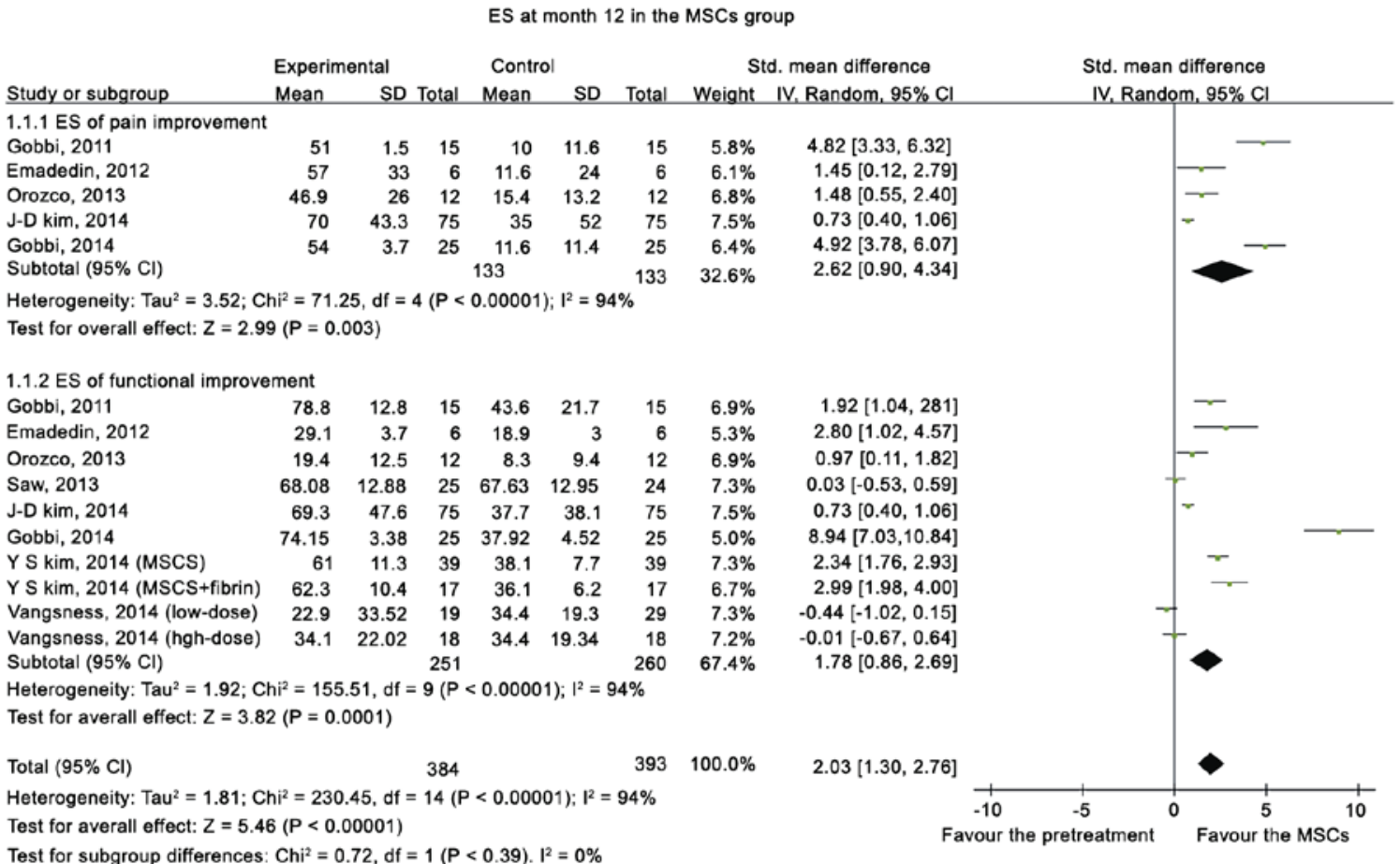

B

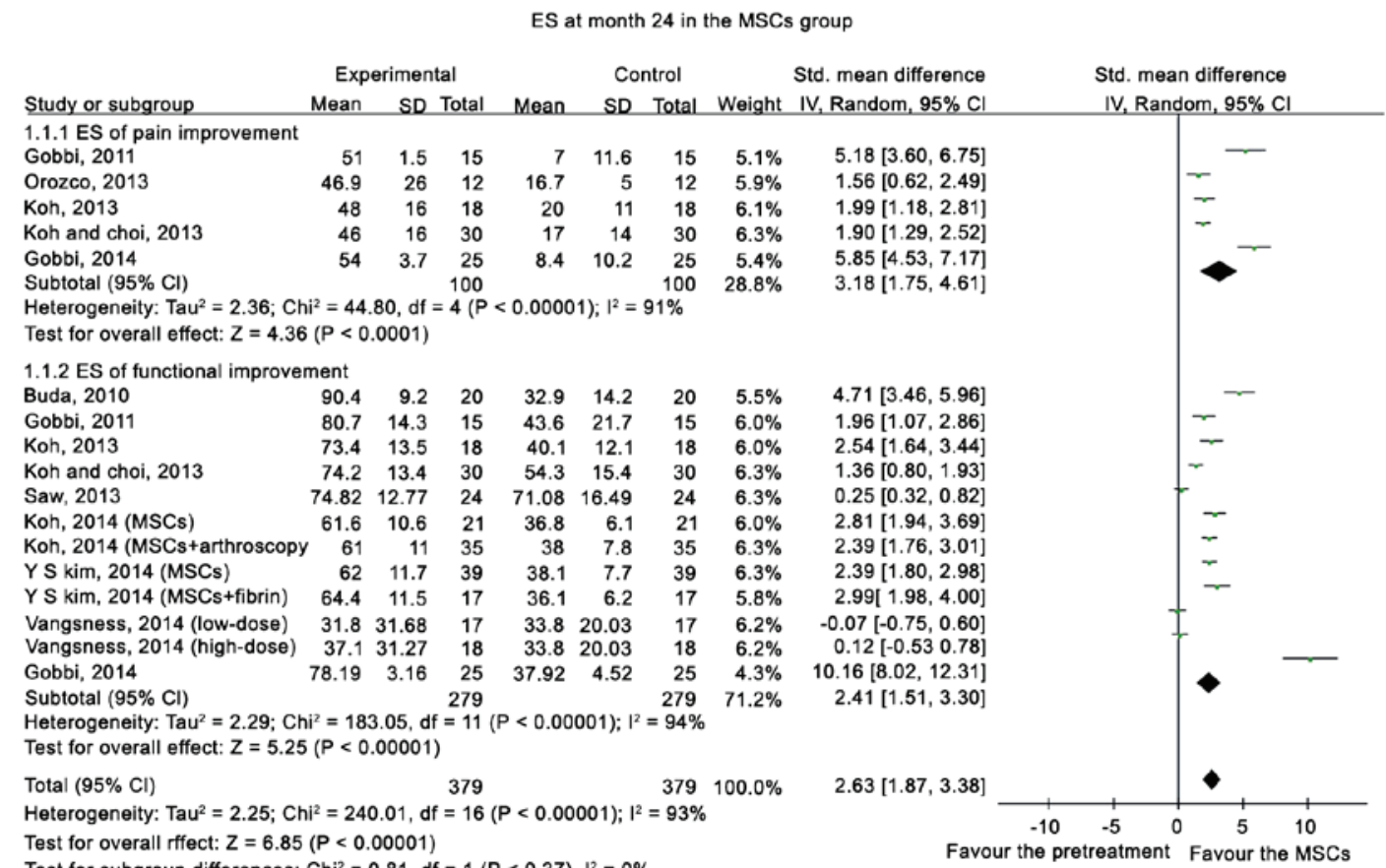

Figure 3. Forest plot of ES of pain and functional changes from baseline at (A) 12 and (B) 24 months after MSC treatment. ES, effect size; MSC, mesenchymal stem cell; SD, standard deviation; IV, inverse variance; CI, confidence interval.

Following analysis of the pooled ESs at 12 and 24 months, these values were higher than the summed ESs at 3 months, which indicated that the treatment effect of MSCs did not decrease in a time-dependent manner. However, a dose-responsiveness association was not demonstrated in the MSC numbers. The treatment effectiveness in the MSC groups treated with AD or activation agents was superior to the MSCs groups alone. Notably, the early OA group exhibited a higher ES point estimate at all time points, as compared with the advanced OA group.

To the best of our knowledge, no previous meta-analytic research has quantified the effectiveness of MSC treatment and analyzed the factors and modified the outcomes. Several reviews of the literature (35-38) have analyzed the role of MSCs therapy in KOA. Barry and Murphy (37) stressed that paracrine factor must be used as a measure to evaluate the 
Table III. Analysis of the effect sizes of MSC treatment stratified by the indicated subgroups.

\begin{tabular}{|c|c|c|c|c|}
\hline Subgroup & $\begin{array}{c}\text { Pooled effect } \\
\text { size at month } 3\end{array}$ & $\begin{array}{c}\text { Pooled effect } \\
\text { size at month } 6\end{array}$ & $\begin{array}{c}\text { Pooled effect } \\
\text { size at month } 12\end{array}$ & $\begin{array}{c}\text { Pooled effect } \\
\text { size at month } 24\end{array}$ \\
\hline \multicolumn{5}{|l|}{ Study design } \\
\hline Single-arm follow-up study & $0.48(0.18-0.77)$ & $1.48(0.51-2.44)$ & $2.66(1.69-3.62)$ & $2.87(1.99-3.75)$ \\
\hline Quasi-experimental study & $0.75(0.17-1.32)$ & $1.37(0.59-2.14)$ & $2.53(1.96-3.10)$ & $2.53(2.18-2.89)$ \\
\hline Randomized controlled trial & $1.87(1.19-2.54)$ & $1.09(-0.35-2.53)$ & $0.14(0.49-0.20)$ & $0.12(0.24-0.48)$ \\
\hline \multicolumn{5}{|l|}{ MSCs doses administered } \\
\hline$<5 \times 10^{6}$ & $0.34(-0.08-0.75)$ & 0.70 (0.46-0.93) & $1.60(0.73-2.46)$ & $2.25(1.54-2.97)$ \\
\hline $5 \times 10^{6}-5 \times 10^{7}$ & $0.89(0.36-1.42)$ & $1.39(0.80-1.99)$ & $1.60(0.55-2.65)$ & $-0.07(-0.75-0.60)$ \\
\hline$>1 \times 10^{7}$ & $0.67(0.09-1.26)$ & $1.91(0.58-3.23)$ & $-0.01(-0.67-0.64)$ & $0.12(-0.53-0.78)$ \\
\hline \multicolumn{5}{|l|}{ Arthroscopic debridement } \\
\hline Yes & $0.37(0.01-0.74)$ & $0.45(-0.16-1.06)$ & $2.20(1.30-3.09)$ & $2.32(1.61-3.03)$ \\
\hline No & $1.02(0.58-1.47)$ & $1.48(0.80-2.16)$ & $1.41(0.83-2.00)$ & $1.56(0.62-2.49)$ \\
\hline \multicolumn{5}{|l|}{ Activation agent } \\
\hline Yes & $0.37(0.01-0.74)$ & $1.40(0.26-2.54)$ & $3.13(1.55-4.71)$ & $2.82(2.07-3.56)$ \\
\hline No & $1.02(0.58-1.47)$ & $1.29(0.53-2.05)$ & $0.67(0.01-1.34)$ & $0.84(0.16-1.52)$ \\
\hline \multicolumn{5}{|l|}{ Severity of degeneration } \\
\hline Early OA & $1.55(0.66-2.45)$ & $4.10(3.16-5.04)$ & $2.53(1.96-3.10)$ & $2.53(2.18-2.89)$ \\
\hline Advanced OA & $0.78(0.34-1.22)$ & $2.40(1.34-3.46)$ & $1.99(0.70-3.28)$ & $2.54(1.64-3.44)$ \\
\hline
\end{tabular}

Values are expressed by their point estimates with a 95\% CI. 95\% CI covered a zero value, which indicated an uncertainty of treatment effectiveness compared with the pretreatment baseline. MSC, mesenchymal stem cell; OA, osteoarthritis; CI, confidence interval.

\section{A}

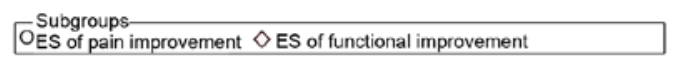

Funnel plot with $95 \%$ confidence limits

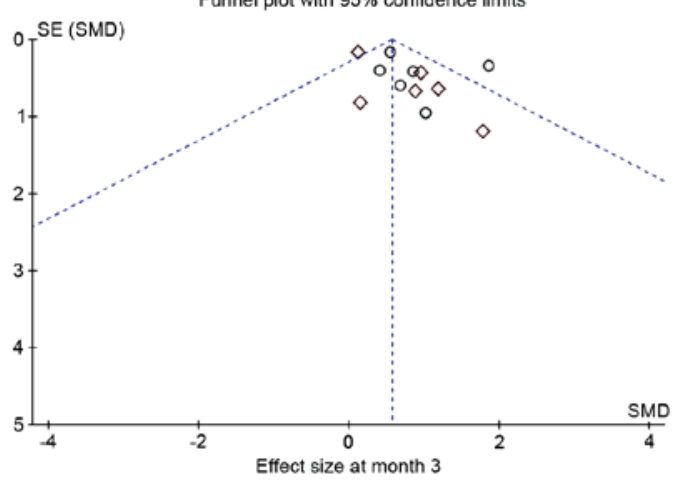

C OES of pain improvement $\triangle \mathrm{ES}$ of functional improvement

Funnel plot with $95 \%$ confidence limits

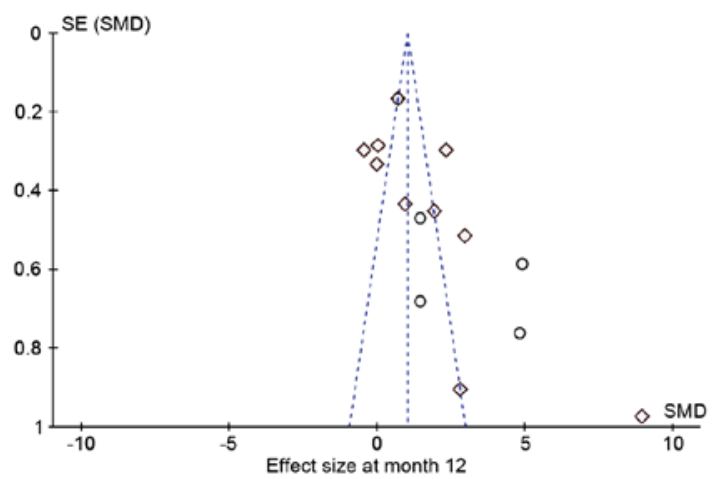

B
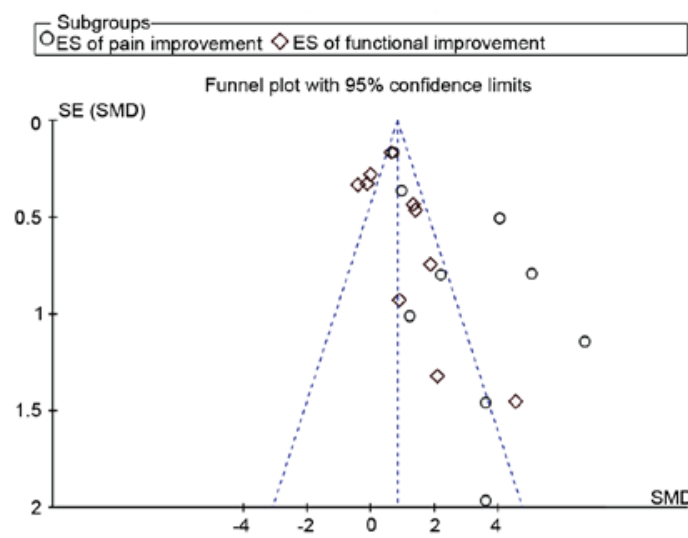

Funnel plot with $95 \%$ confidence limits

D OES of pain improvement $\diamond$ ES of functional improvement

Funnel plot with $95 \%$ confidence limits

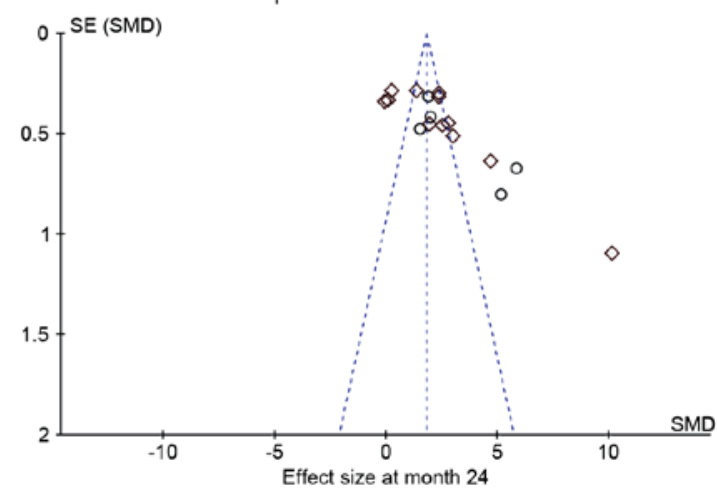

Figure 4. Funnel plots of the ES of pain and functional changes from baseline at (A) 3, (B) 6, (C) 12 and (D) 24 months post-MSC treatment. ES, effect size; MSC, mesenchymal stem cell; SE, standard error; SMD, standard mean difference. 
potential treatment of MSCs in order to replace traditional measures based on differentiation and cell-surface markers. They also outlined that early-stage clinical trials are underway for test the method of intra-articular injection of MSCs into the knee. However, the optimal dose and vehicle have not been established. Filardo et al (38) reported that, due to the prevalence of low-quality preclinical studies and clinical trials, knowledge on the treatment of MSCs for cartilage regeneration remains preliminary, despite the growing interest in the biological approach. Rodriguez-Merchan (35) highlighted the efficacy of utilizing intra-articular injections of MSCs to treat KOA; however, the results of the treatment are simply encouraging. Kristjansson and Honsawek (36) discussed and assessed three ways in which MSCs may be used to treat OA patients by intra-articular injections and implantation as well as micro fracture. They reported that with higher numbers of MSCs injected superior results would be obtained. However, in order to facilitate the treatment, a single injection of MSCs alone or in combination of growth factors would be the ultimate solution.

The present meta-analysis suggested that MSC treatment significantly improved pain and functional status, relative to the basal evaluations in KOA, and the beneficial effect was maintained for two years after treatment. Furthermore, the treatment effectiveness did not reduce over time. Several factors mentioned by anecdotal research may modify the ESs of MSC treatment. In terms of the study design, the pooled ESs in single-arm and quasi-experimental studies were likely to be higher than those in RCTs. However, the results of these RCT studies suggested that MSCs also reduce pain and improve function in patients with KOA. Regarding the number of MSCs used in treatment, a dose-responsiveness relationship remained unclear. Jo et al (48) enrolled 18 patients who were injected with ADMSCs into the knee. The study consisted of three groups, the low-dose $\left(1.0 \times 10^{7}\right.$ cells $)$, mid-dose $\left(5.0 \times 10^{7}\right)$, and high-dose $\left(1.0 \times 10^{8}\right)$ groups. However, a significant improvement in joint function and reduction in pain was observed in the low and mid-dose groups. Conversely, in previous studies, an increased number of cells yielded superior results. Therefore, the optimal dose and vehicle are yet to be established. One potential modifier is the AD. The present stratified analysis suggested that AD potentially contributed to an increase in treatment effectiveness. Another issue is the addition of activation agents, particularly at 12 months in the activation agents group (ES, 3.13; 95\% CI, 1.55-4.71) compared with the group without activation agents (ES, 0.67; 95\%CI, 0.01-1.34). The present subgroup analysis showed that the efficacy varied according to the degenerative severity, which was associated with the regenerative potential of damaged cartilage. These results are compatible with the findings of the majority of previous trials, and the early OA group exhibited a higher ES point estimated at all time points than the advanced OA group.

\section{Acknowledgements}

The study was supported by grants from the National Science Foundation of China (grant nos. 81160225, 81260453 and 81360451) and the Xinjiang Bingtuan Special Program of Medical Science (grant nos. 2014CC002, 2013BA020 and 2012BC002).

\section{References}

1. Findlay DM: If good things come from above, do bad things come from below? Arthritis Res Ther 12: 119, 2010.

2. Goldring MB and Goldring SR: Articular cartilage and subchondral bone in the pathogenesis of osteoarthritis. Ann NY Acad Sci 1192: 230-237, 2010.

3. Gross JB, Guillaume C, Gégout-Pottie P, Mainard D and Presle N: Synovial fluid levels of adipokines in osteoarthritis: Association with local factors of inflammation and cartilage maintenance. Biomed Mater Eng 24 (Suppl 1): S17-S25, 2014.

4. Hawker GA, Mian S, Bednis K and Stanaitis I: Osteoarthritis year 2010 in review: Non-pharmacologic therapy. Osteoarthritis Cartilage 19: 366-374, 2011.

5. Sakata K, Furumatsu T, Abe N, Miyazawa S, Sakoma Y and Ozaki T: Histological analysis of failed cartilage repair after marrow stimulation for the treatment of large cartilage defect in medial compartmental osteoarthritis of the knee. Acta Med Okayama 67: 65-74, 2013.

6. Lee GW, Son JH, Kim JD and Jung GH: Is platelet-rich plasma able to enhance the results of arthroscopic microfracture in early osteoarthritis and cartilage lesion over 40 years of age? Eur J Orthop Surg Traumatol 23: 581-587, 2013.

7. Eldracher M, Orth P, Cucchiarini M, Pape D and Madry H: Small subchondral drill holes improve marrow stimulation of articular cartilage defects. Am J Sports Med 42: 2741-2750, 2014.

8. Dowsey MM, Gunn J and Choong PF: Selecting those to refer for joint replacement: Who will likely benefit and who will not? Best Pract Res Clin Rheumatol 28: 157-171, 2014.

9. Knutsen G, Drogset JO, Engebretsen L, Grøntvedt T, Isaksen V, Ludvigsen TC, Roberts S, Solheim E, Strand T and Johansen O: A randomized trial comparing autologous chondrocyte implantation with microfracture. Findings at five years. J Bone Joint Surg Am 89: 2105-2112, 2007.

10. Lee CR, Grodzinsky AJ, Hsu HP, Martin SD and Spector M: Effects of harvest and selected cartilage repair procedures on the physical and biochemical properties of articular cartilage in the canine knee. J Orthop Res 18: 790-799, 2000.

11. Vasiliadis HS and Wasiak J: Autologous chondrocyte implantation for full thickness articular cartilage defects of the knee. Cochrane Database Syst Rev (10): CD003323, 2010.

12. Mandelbaum B, Browne JE, Fu F, Micheli LJ, Moseley JB Jr, Erggelet $C$ and Anderson AF: Treatment outcomes of autologous chondrocyte implantation for full-thickness articular cartilage defects of the trochlea. Am J Sports Med 35: 915-921, 2007.

13. Phinney DG and Prockop DJ: Concise review: Mesenchymal stem/multipotent stromal cells: The state of transdifferentiation and modes of tissue repair-current views. Stem cells 25: 2896-2902, 2007.

14. Delorme B, Ringe J, Pontikoglou C, Gaillard J, Langonné A, Sensebé L, Noël D, Jorgensen C, Häupl T and Charbord P: Specific lineage-priming of bone marrow mesenchymal stem cells provides the molecular framework for their plasticity. Stem Cells 27: 1142-1151, 2009.

15. Oreffo RO, Cooper C, Mason C and Clements M: Mesenchymal stem cells: Lineage, plasticity and skeletal therapeutic potential. Stem Cell Rev 1: 169-178, 2005.

16. Robey PG and Bianco P: The use of adult stem cells in rebuilding the human face. J Am Dent Assoc 137: 961-972, 2006.

17. Caplan AI: Why are MSCs therapeutic? New data: New insight. J Pathol 217: 318-324, 2009.

18. Davatchi F, Sadeghi Abdollahi B, Mohyeddin M and Nikbin B: Mesenchymal stem cell therapy for knee osteoarthritis: 5 years follow-up of three patients. Int J Rheum Dis, 2015.

19. Emadedin M, Aghdami N, Taghiyar L, Fazeli R, Moghadasali R, Jahangir S, Farjad R and Baghaban Eslaminejad M: Intra-articular injection of autologous mesenchymal stem cells in six patients with knee osteoarthritis. Arch Iran Med 15: 422-428, 2012.

20. Koh YG, Jo SB, Kwon OR, Suh DS, Lee SW, Park SH and Choi YJ: Mesenchymal stem cell injections improve symptoms of knee osteoarthritis. Arthroscopy 29: 748-755, 2013.

21. Higgins JP, Thompson SG, Deeks JJ and Altman DG: Measuring inconsistency in meta-analyses. BMJ 327: 557-560, 2003.

22. Kuroda R, Ishida K, Matsumoto T, Akisue T, Fujioka H, Mizuno K, Ohgushi H, Wakitani S and Kurosaka M: Treatment of a full-thickness articular cartilage defect in the femoral condyle of an athlete with autologous bone-marrow stromal cells. Osteoarthritis Cartilage 15: 226-231, 2007. 
23. Wakitani S, Nawata M, Tensho K, Okabe T, Machida $\mathrm{H}$ and Ohgushi H: Repair of articular cartilage defects in the patello-femoral joint with autologous bone marrow mesenchymal cell transplantation: Three case reports involving nine defects in five knees. J Tissue Eng Regen Med 1: 74-79, 2007.

24. Centeno CJ, Busse D, Kisiday J, Keohan C, Freeman M and Karli D: Increased knee cartilage volume in degenerative joint disease using percutaneously implanted, autologous mesenchymal stem cells. Pain Physician 11: 343-353, 2008.

25. Centeno CJ, Busse D, Kisiday J, Keohan C, Freeman M and Karli D: Regeneration of meniscus cartilage in a knee treated with percutaneously implanted autologous mesenchymal stem cells. Med Hypotheses 71: 900-908, 2008.

26. Wakitani S, Mitsuoka T, Nakamura N, Toritsuka Y, Nakamura Y and Horibe S: Autologous bone marrow stromal cell transplantation for repair of full-thickness articular cartilage defects in human patellae: Two case reports. Cell Transplant 13: 595-600, 2004.

27. Wakitani S, Imoto K, Yamamoto T, Saito M, Murata N and Yoneda M: Human autologous culture expanded bone marrow mesenchymal cell transplantation for repair of cartilage defects in osteoarthritic knees. Osteoarthritis Cartilage 10: 199-206, 2002.

28. Nejadnik H, Hui JH, Feng Choong EP, Tai BC and Lee EH: Autologous bone marrow-derived mesenchymal stem cells versus autologous chondrocyte implantation: An observational cohort study. Am J Sports Med 38: 1110-1116, 2010.

29. Wakitani S, Okabe T, Horibe S, Mitsuoka T, Saito M, Koyama T, Nawata M, Tensho K, Kato H and Uematsu K: Safety of autologous bone marrow-derived mesenchymal stem cell transplantation for cartilage repair in 41 patients with 45 joints followed for up to 11 years and 5 months. J Tissue Eng Regen Med 5: 146-150, 2011

30. Saw KY, Anz A, Merican S, Tay YG, Ragavanaidu K, Jee CS and McGuire DA: Articular cartilage regeneration with autologous peripheral blood progenitor cells and hyaluronic acid after arthroscopic subchondral drilling: A report of 5 cases with histology. Arthroscopy 27: 493-506, 2011.

31. Hauser RA and Orlofsky A: Regenerative injection therapy with whole bone marrow aspirate for degenerative joint disease: A case series. Clin Med Insights Arthritis Musculoskelet Disord 6 : 65-72, 2013.

32. Orozco L, Munar A, Soler R, Alberca M, Soler F, Huguet M, Sentís J, Sánchez A and García-Sancho J: Treatment of knee osteoarthritis with autologous mesenchymal stem cells: Two-year follow-up results. Transplantation 97: e66-e68, 2014

33. Centeno CJ, Schultz JR, Cheever M, Freeman M, Faulkner S, Robinson B and Hanson R: Safety and complications reporting update on the re-implantation of culture-expanded mesenchymal stem cells using autologous platelet lysate technique. Curr Stem Cell Res Ther 6: 368-378, 2011.

34. Centeno CJ, Schultz JR, Cheever M, Robinson B, Freeman M and Marasco W: Safety and complications reporting on the re-implantation of culture-expanded mesenchymal stem cells using autologous platelet lysate technique. Curr Stem Cell Res Ther 5: 81-93, 2010

35. Rodriguez-Merchán EC: Intra-articular injections of mesenchymal stem cells for knee osteoarthritis. Am J Orthop (Belle Mead NJ) 43: E282-E291, 2014.

36. Kristjnsson B and Honsawek S: Current perspectives in mesenchymal stem cell therapies for osteoarthritis. Stem Cells Int 2014: 194318, 2014

37. Barry F and Murphy M: Mesenchymal stem cells in joint disease and repair. Nat Rev Rheumatol 9: 584-594, 2013.

38. Filardo G, Madry H, Jelic M, Roffi A, Cucchiarini M and Kon E: Mesenchymal stem cells for the treatment of cartilage lesions: From preclinical findings to clinical application in orthopaedics. Knee Surg Sports Traumatol Arthrosc 21: 1717-1729, 2013.
39. Buda R, Vannini F, Cavallo M, Grigolo B, Cenacchi A and Giannini S: Osteochondral lesions of the knee: A new one-step repair technique with bone-marrow-derived cells. J Bone Joint Surg Am 92 (Suppl 2): S2-S11, 2010.

40. Gobbi A, Karnatzikos G, Scotti C, Mahajan V, Mazzucco L and Grigolo B: One-step cartilage repair with bone marrow aspirate concentrated cells and collagen matrix in full-thickness knee cartilage lesions: Results at 2 year follow-up. Cartilage 2: 286-299, 2011.

41. Turajane T, Chaweewannakorn U, Larbpaiboonpong V, Aojanepong J, Thitiset T, Honsawek S, Fongsarun J and Papadopoulos KI: Combination of intra-articular autologous activated peripheral blood stem cells with growth factor addition/ preservation and hyaluronic acid in conjunction with arthroscopic microdrilling mesenchymal cell stimulation improves quality of life and regenerates articular cartilage in early osteoarthritic knee disease. J Med Assoc Thai 96: 580-588, 2013.

42. Orozco L, Munar A, Soler R, Alberca M, Soler F, Huguet M, Sentís J, Sánchez A and García-Sancho J: Treatment of knee osteoarthritis with autologous mesenchymal stem cells: A pilot study. Transplantation 95: 1535-1541, 2013.

43. Kim JD, Lee GW, Jung GH, Kim CK, Kim T, Park JH, Cha SS and You YB: Clinical outcome of autologous bone marrow aspirates concentrate (BMAC) injection in degenerative arthritis of the knee. Eur J Orthop Surg Traumatol 24: 1505-1511, 2014.

44. Koh YG, Choi YJ, Kwon SK, Kim YS and Yeo JE: Clinical results and second-look arthroscopic findings after treatment with adipose-derived stem cells for knee osteoarthritis. Knee Surg Sports Traumatol Arthrosc 23: 1308-1316, 2015.

45. Gobbi A, Karnatzikos G and Sankineani SR: One-step surgery with multipotent stem cells for the treatment of large full-thickness chondral defects of the knee. Am J Sports Med 42: 648-657, 2014.

46. Koh YG and Choi YJ: Infrapatellar fat pad-derived mesenchymal stem cell therapy for knee osteoarthritis. Knee 19: 902-907,2012.

47. Koh YG, Choi YJ, Kwon OR and Kim YS: Second-look arthroscopic evaluation of cartilage lesions after mesenchymal stem cell implantation in osteoarthritic knees. Am J Sports Med 42: 1628-1637, 2014.

48. Jo CH, Lee YG, Shin WH, Kim H, Chai JW, Jeong EC, Kim JE, Shim H, Shin JS, Shin IS, et al: Intra-articular injection of mesenchymal stem cells for the treatment of osteoarthritis of the knee: A proof-of-concept clinical trial. Stem Cells 32: 1254-1266, 2014

49. Kim YS, Choi YJ, Suh DS, Heo DB, Kim YI, Ryu JS and Koh YG: Mesenchymal stem cell implantation in osteoarthritic knees: Is fibrin glue effective as a scaffold? Am J Sports Med 43: 176-185, 2015.

50. Varma HS, Dadarya B and Vidyarthi A: The new avenues in the management of osteo-arthritis of knee-stem cells. J Indian Med Assoc 108: 583-585, 2010

51. Saw KY, Anz A, Siew-Yoke JC, Merican S, Ching-Soong Ng R, Roohi SA and Ragavanaidu K: Articular cartilage regeneration with autologous peripheral blood stem cells versus hyaluronic acid: A randomized controlled trial. Arthroscopy 29: 684-694, 2013.

52. Wong KL, Lee KB, Tai BC, Law P, Lee EH and Hui JH: Injectable cultured bone marrow-derived mesenchymal stem cells in varus knees with cartilage defects undergoing high tibial osteotomy: A prospective, randomized controlled clinical trial with 2 years follow-up. Arthroscopy 29: 2020-2028, 2013.

53. VangsnessCT Jr, Farr J II, Boyd J, Dellaero DT, Mills CR and LeRoux-Williams M: Adult human mesenchymal stem cells delivered via intra-articular injection to the knee following partial medial meniscectomy: A randomized, double-blind, controlled study. J Bone Joint Surg Am 96: 90-98, 2014 